\title{
5. Komplementärmedizin in der Psychiatrie
}

\author{
HANS-WOLFGANG HOEFERT
}

\section{Einleitung}

Zu den psychischen Störungen zählen sowohl manifeste Erkrankungen wie Depression und Schizophrenie, neurogenerative Erkrankungen wie bei der Demenz, aber auch Störungen der emotionalen Befindlichkeit wie z.B. bei diversen Angststörungen. Ein Teil dieser Störungen wird genetisch begründet (insbesondere Psychosen), ein anderer Teil (insbesondere Angst-, Zwangs- und Essstörungen) eher betrachtet als Resultat einer ungünstigen Lerngeschichte oder einer ungünstigen sozialen Umwelt. Substanzabhängigkeit wird auf genetische Dispositionen in der Interaktion mit bestimmten Bedingungen zurückgeführt. Für die Komplementärmedizin ergeben sich im Rahmen dieses Störungsspektrums unterschiedliche Ansatzpunkte für Interventionen, welche einerseits direkt in die Körperphysiologie eingreifen, andererseits eher indirekt die Einstellung zum eigenen Körper und den Umgang mit psychischen Spannungen und Konflikten beeinflussen.

\subsection{Epidemiologie von psychischen Störungen und Inanspruchnahme von KM}

Nach der jüngsten zur psychischen Gesundheit Erwachsener in Deutschland (DEGS) litten mit einer 12-Monatsprävalenz etwa 33\% der Bevölkerung unter einer oder mehreren psychischen Störungen. Die häufigsten Störungen bei Männern waren Substanzstörungen, gefolgt von Angststörungen und Depressionen, bei 
Frauen Angststörungen, gefolgt von Depressionen und somatoformen Störungen. Die höchsten Prävalenzraten lagen in der Altersgruppe von 18-34 Jahren (Wittchen \& Jacobi 2012), wobei Depressionen eher im höheren Alter bei den 6o-69Jährigen auftreten als in jüngeren Altersgruppen (Busch et al. 2013). Derartige Störungen tragen nicht nur zu einer Verminderung der Lebensqualität und $\mathrm{zu}$ Arbeitsausfällen bei, sondern werden auch nur in der Hälfte der Fälle ausreichend versorgt, sofern sie überhaupt diagnostiziert werden (Gaebel 2012). Statistisch nicht erfasst werden zumeist Befindlichkeitsstörungen, die mit dem Globalbegriff „Stress“ versehen werden und sich eher indirekt (z.B. als Schlafstörungen oder depressive Verstimmungen) bemerkbar machen. Frauen klagen häufiger über Stress-bedingte Störungen als Männer, wobei sich soziale Unterstützung und ein höherer sozioökonomischer Status stressmindernd auszuwirken scheint (Hapke et al. 2013).

Psychische Störungen werden in deutschen Hausarztpraxen bei einem Viertel der Patienten diagnostiziert; gleichzeitig ist der Hausarzt die erste Anlaufstelle bei psychischen Problemen - noch vor den Fachärzten, Psychiatern und Psychotherapeuten, wobei die Patienten in der Regel eine konventionelle medizinische Versorgung erhalten (Harfst \& Marstedt 2009). Nach den Daten aus dem letzten Bundesgesundheitssurvey von 1998 (Zusatzbefragung nach psychischen Problemen) besteht bei einem Drittel der Bevölkerung ein Bedarf nach entsprechender Beratung oder Behandlung. Am häufigsten vertreten sind - in dieser Reihenfolge - Angststörungen, Substanzabhängigkeit (davon am häufigsten Alkohol), Depressionen und somatoforme Störungen (Jacobi \& Harfst 2007). Entsprechend verzeichnen die Krankenkassen ein Anwachsen von Ausfallzeiten aufgrund von psychischen Störungen. Jene sind seit den goer-Jahren als Krankheitsursache vom 5. auf den 3. Platz gerückt und stellen auch eine Ursache für Frühverrentungen dar. Im Einzelnen ist eine Zunahme von Depressionen insbesondere bei Frauen und von Substanzabhängigkeit bei Männern zu vermerken (bei der Schizophrenie ist das Geschlechterverhältnis ausgewogen). Diese Zunahme spiegelt sich u.a. in der deutlich gestiegenen Verschreibung von Antidepressiva wider (Lademann et al. 2006). Beachtenswert ist schließlich, dass auch bei Kindern und Jugendlichen psychische Störungen - nach Asthma, Allergien und Adipositas auf dem vierten Platz rangieren; unter diesen sind Angststörungen, Depressionen, Aufmerksamkeits-Defizitstörungen und Störungen des Sozialverhaltens am häufigsten vertreten (Ravens-Sieberer et al. 2007).

Viele Patienten mit psychischen Störungen nutzen Verfahren und Produkte der Komplementärmedizin (KM) in Eigenregie und informieren auch nur selten ihren Arzt darüber. Da ein Teil dieser Produkte frei verkäuflich ist, sind Fehldosierungen und Interaktionen mit parallel eigenommenen herkömmlichen Medikamenten nicht auszuschließen. So ist es auch nicht erstaunlich, dass diejenigen Patienten, welche sich wegen ihrer psychischen Störungen in eine ambulante oder stationäre Behandlung begeben, bereits Erfahrungen im Umgang mit Verfahren oder Produkten der KM haben. Aus der Befragung von stationären Patien- 
ten durch Huber et al. (2004) geht hervor, dass 26\% bereits bei einem Arzt mit KM-Orientierung und 19\% bereits bei einem Heilpraktiker waren. Etwa je ein Fünftel hatten Erfahrungen mit körperlichen Verfahren, Diät, Massage und Vitaminen bzw. Nahrungszusätzen; jeder zehnte Patient hatte Erfahrungen mit Heilkräutern, Akupunktur und Homöopathie. Die höchste KM-Nutzung war bei Patienten der Krankenhausabteilung Psychosomatik zu finden, danach in der Gastroenterologie, Onkologie und Kardiologie. Ein Viertel der Patienten glaubten, dass ihnen die KM helfen könne. Als Prädiktoren für eine KM-Präferenz nennen die Autoren junges Alter, Aversion gegen „chemische“ Medikamente, Wunsch nach Mitbeteiligung beim Therapieregime, die Motivation zur Veränderung des eigenen Lebensstils und das Interesse an einer „ganzheitlichen“ Behandlung. In einer vergleichbaren australischen Krankenhausstudie (Alderman et al. 2003) nutzten die Patienten hauptsächlich Vitamine, Mineralien und Heilkräuter, wobei sie damit zufriedener waren als mit konventionellen Medikamenten.

Werneke et al. (2006) resümieren, dass von den - stationären und nichtstationären - Patienten mit psychischen Störungen je nach Studie zwischen 8 und 57\% der Patienten bereits KM-Verfahren genutzt haben, am häufigsten Patienten mit Angst- bzw. Panikstörungen und Depressionen (zum Teil auch im Zusammenhang mit Krebserkrankungen). Als Motive gaben sie vor allem an: Dämpfung der Nebenwirkungen von konventionellen Medikamenten, Ineffektivität einer konventionellen Behandlung und Bevorzugung eines „ganzheitlichen“ Ansatzes. Auch Russinova et al. (2002) hatten Patienten mit psychischen Störungen danach befragt, was sie sich von der KM-Nutzung erhoffen. Am häufigsten wurden genannt (in dieser Reihenfolge): größere emotionale Stabilität, verbesserte Konzentration, mehr innere Stärke und mehr allgemeines Wohlbefinden.

Bei allen der hier diskutierten psychischen Störungen ist zu bedenken, dass es sich zumindest im Ansatz immer um Möglichkeiten der menschlichen Gefühlsvariation handelt (wenn man einmal von neurologischen Degenerationsprozessen absieht), welche erst auf dem Wege der klinischen Diagnostik einen Krankheitswert erhalten. Aber auch die Patienten selbst tragen dazu bei, dass ihre Gefühlswahrnehmungen zu klinischen Symptomen werden, indem sie ihren Gesundheitszustand kritisch beobachten und entsprechende Gesundheitsängste (vgl. Hoefert \& Klotter 2012) entwickeln.

Therapeutisch bedeutsam ist die Beobachtung, dass die meisten Menschen angemessen mit Gefühlsirritationen und auch passageren Gefühlsstörungen umgehen können und, falls überhaupt, KM-Methoden niedrigdosiert nutzen; ein Problem entsteht erst, wenn KM-Methoden z.B. bei Ängsten und Depressionen längerfristig und in Kombination mit verordneten Psychopharmaka zum Einsatz kommen (Ravindran \& Sarris 2013)

Im Folgenden wird die mögliche Bedeutung von KM bei typischen Diagnosegruppen der Psychiatrie näher erläutert. Zusätzlich wird die Demenz als typische Indikation in der Geriatrie in diese Übersicht aufgenommen. Bei der Darstellung der für eine Behandlung mehr oder weniger geeigneten KM-Verfahren bleiben 
neben den konventionellen Medikamenten (Psychopharmaka) auch Verfahren, die eher zur psychologischen Psychotherapie bzw. zur kognitiven Verhaltenstherapie zu rechnen sind, außer Betracht.

\subsection{Klassische Indikationen}

\subsubsection{Angst}

Angststörungen präsentieren sich habituell als generalisierte Angststörung oder sind objekt- oder situationsgebunden (spezifische Ängste bzw. Phobien); oftmals sind sie mit Depressionen konfundiert. Zur Therapie von Angststörungen werden in der konventionellen Medizin bzw. Psychiatrie und Psychotherapie Anxiolytika und Sedativa bzw. kognitive Verhaltenstherapie eingesetzt. Aber auch die KM kann hier einen therapeutischen Beitrag leisten, wobei die Wirkung von KMMethoden größtenteils darauf beruht, dass belastende oder intrusive Gedanken verringert und die damit einhergehende körperliche Anspannung reduziert wird (s. Tab. 17). In einer neueren US-amerikanischen Studie (Bystritsky et al. 2012) zeigte sich, dass mehr als $40 \%$ aller Angst-Patienten bereits Erfahrungen mit Mitteln und Methoden der KM hatten und deshalb eine Interaktion zwischen konventionellen Pychopharmaka und KM-Produkten nicht ausgeschlossen werden kann.

Tab. 17 Bewährung von KM-Methoden bei Angststörungen

\begin{tabular}{|c|c|c|}
\hline Methode & Ergebnis in Kurzform & Quelle \\
\hline Methodenvergleich & $\begin{array}{l}\text { hohe Evidenz für Kava (piper methysticum) } \\
\text { gewisse Evidenz für Inositol, Tanz und Bewegungstherapie, } \\
\text { Gymnastik und Entspannung } \\
\text { keine Evidenz für Gingko biloba, Baldrian, ind. Wassernabel, } \\
\text { Bachblüten und Vitamine }\end{array}$ & Jorm et al. 2004 \\
\hline Methodenvergleich & $\begin{array}{l}\text { hohe Evidenz für Kava (Piper methysticum) } \\
\text { gewisse Evidenz für Inositol } \\
\text { keine Evidenz für Baldrian (Vareliana off.) }\end{array}$ & Ernst 2007 \\
\hline Methodenvergleich & $\begin{array}{l}\text { hohe Evidenz für Kava (Piper methysticum) } \\
\text { gewisse Evidenz für Inositol } \\
\text { keine Evidenz für Cannabis, Omega-3-Fettsäuren und generell } \\
\text { Vitamine }\end{array}$ & Saeed et al. 2007 \\
\hline Methodenvergleich & $\begin{array}{l}\text { positive Effekte für Bewegung und Yoga } \\
\text { Tai Chi, Qigong und Meditation nicht effektiv }\end{array}$ & Saeed et al. 2010 \\
\hline Methodenvergleich & nur geringe Evidenz für Meditation & Williams et al. 2011 \\
\hline
\end{tabular}




\begin{tabular}{|c|c|c|}
\hline Methode & Ergebnis in Kurzform & Quelle \\
\hline & $\begin{array}{l}\text { Entspannungs- und Atemtechniken vielversprechend für Panikstö- } \\
\text { rungen } \\
\text { Achtsamkeitsbasierte Stress-Reduktion vielversprechend bei } \\
\text { Angst- und Depressionssysymptomen }\end{array}$ & \\
\hline Methodenvergleich & Bewegungsübungen mittel effektiv für Angst- und Panikstörungen & $\begin{array}{l}\text { Ravindran \& de } \\
\text { Silva } 2013\end{array}$ \\
\hline Meditation & $\begin{array}{l}\text { nur geringer Einfluss auf Angstminderung } \\
\text { beeinflusst nur Angstsymptome, aber nicht Angststörung } \\
\text { mögliche Verbesserung durch Achtsamkeitsmeditation, sofern } \\
\text { nicht nur Entspannungseffekte, sondern auch kognitive Umstruk- } \\
\text { turierungen und eine andere Verarbeitung von Emotionen er- } \\
\text { reicht wird }\end{array}$ & $\begin{array}{l}\text { Krisanaprakornit et } \\
\text { al. } 2009 \\
\text { Chen et al. } 2012 \\
\text { Edenfeld \& Saeed } \\
2012\end{array}$ \\
\hline Qigong & $\begin{array}{l}\text { kein Einfluss auf Angstminderung, geringer Einfluss bei Depressi- } \\
\text { on }\end{array}$ & Wang et al. 2013 \\
\hline Phytopharmaka & $\begin{array}{l}\text { Johanniskraut, Baldrian oder Inositol werden in ihrer Wirksamkeit } \\
\text { häufig überschätzt } \\
\text { Lavendel (oral verabreicht) scheint einen gewissen therapeuti- } \\
\text { schen Effekt zu haben }\end{array}$ & $\begin{array}{l}\text { Saeed et al. } 2007 \\
\text { Perry et al. } 2012\end{array}$ \\
\hline Hypnose & einige Studien lassen Bewährung bei habitueller Angst erkennen & Hammond 2010 \\
\hline Akupunktur & insbesondere Ohrakupunktur zeigt tendenziell positive Wirkungen & $\begin{array}{l}\text { Pilkington et al. } \\
2007\end{array}$ \\
\hline Aromatherapie & $\begin{array}{l}\text { Effektivität wegen der verschiedenen daran beteiligten Elemente } \\
\text { (Öle, Hautbehandlung, Gerüche) schwer einzuschätzen, aber } \\
\text { wegen der geringen Nebenwirkungen in Betracht zu ziehen }\end{array}$ & $\begin{array}{l}\text { van der Watt } 2008 \\
\text { Lee et al. } 2011\end{array}$ \\
\hline Homöopathie & aufgrund der Studienlage ohne Empfehlung & $\begin{array}{l}\text { Pilkington et al. } \\
2006 \\
\text { Sarris et al. } 2012\end{array}$ \\
\hline
\end{tabular}

Kritisch muss angemerkt werden, dass in den entsprechenden Studien häufig nicht die Spezifität von Angststörungen ausreichend berücksichtigt wird: So haben sich Gymnastik, Meditation, Musik und Entspannung bei einer generalisierten Angststörung bewährt, während Bibliotherapie nur bei spezifischen Phobien oder Tanz- und Bewegungstherapie nur bei Testangst wirksam zu sein scheinen. Einzel-Reviews beziehen sich beispielsweise auf Kava (Pittler \& Ernst 2003, Sarris et al. 2009), aus denen auch das Risiko toxischer Nebenwirkungen erkennbar wird, auf Gymnastik (Martinsen 2008, Larun et al. 2009), auf Yoga (Kirkwood et al. 2005). 
Insgesamt halten Meeks et al. (2007) Mind-Body- und Body-Methoden gegenüber biologisch basierten Methoden für wirksamer. Unter den biologischen Methoden wird in dem neueren Review von Sarris et al. (2012) einzig Kava als wirksam genannt (Kava-Präparate sind allerdings in Deutschland wegen der Lebertoxizität nicht mehr im Handel). Die Studien zu Akupunktur, Yoga und Tai Chi würden eine tendenzielle Evidenz zeigen, während sich die größte Evidenz bei Studien zu Lebensstil-Modifikationen (moderate Bewegung, Achtsamkeitsmeditaton, Reduzierung von Genussmitteln, gesündere Ernährung) zeigten.

Vergleicht man schließlich die Studienlage zur Effektivität von KM-Methoden bei Ängsten mit derjenigen bei Depression, dann zeigt sich, dass sie für Depression eindeutiger ist als für Angst und dadurch gezieltere Empfehlungen zulässt (Ravindran \& de Silva 2013).

\subsubsection{Depression}

Die Prävalenz von Depressionen (ungeachtet ihres Typs, ihrer Dauer und ihres Schweregrades) wird weltweit von der WHO auf 10,4\% geschätzt. In Deutschland beträgt die Lebenszeit-Prävalenz etwa 10\%, die Ein-Jahres-Prävalenz 8\% für Frauen und $4 \%$ für Männer mit den höchsten Werten in der Altersgruppe der 45 - bis 64-Jährigen. Die Betroffenen bemühen sich auf unterschiedliche Weise um die Linderung ihres Leidens, die von der Einnahme von meist pflanzlichen OTCProdukten über verordnete Psychopharmaka bis hin zur Inanspruchnahme einer Psychotherapie reicht. Insbesondere bei einer leichten bis mittelschweren Depression wird gern auf KM-Verfahren zurückgegriffen bzw. werden diese ärztlicherseits empfohlen (vgl. Freeman et al. 2010).

Nach einem Review von Wu et al. (2007) zum Nutzungsverhalten von (weiblichen) depressiven Patienten haben 26\% der Befragten positive Erfahrungen mit manuellen Therapien, 20\% mit Heilkräutern und 16\% mit Vitaminen und Nahrungszusätzen gesammelt. Als Gründe gaben sie an: wenig Nebenwirkungen (45\%), Versagen der konventionellen Medizin (43\%) und direkte Empfehlung des Arztes (33\%). Gleichzeitig betonten sie, dass ihre Nutzung durch den „natürlichen“ Problemzugang, aufgrund der Übereinstimmung mit eigenen Überzeugungen, die Vertrautheit mit KM aus der eigenen Familiengeschichte und durch Medien motiviert sei. Eine Präferenz für KM-Methoden scheint (in den USA) vor allem bei Patienten mit Ängsten bzw. Panikstörungen und Depressionen zu bestehen; diese Patienten halten KM-Verfahren für gleich hilfreich wie konventionelle Medikamente (Kessler et al. 2001).

In der Regel werden KM-Verfahren beim Vorliegen einer Major Depression und seltener in beiden Phasen einer bipolaren Depression (mit depressiven und manischen Phasen) angewendet bzw. untersucht. Die Bewährung von KM-Verfahren nach den Maßstäben der evidenzbasierten Medizin kann anhand von größeren Reviews eingeschätzt werden (s. Tab. 18). Zwar präferieren insbesondere Patienten mit Ängsten und Depressionen häufig KM-Verfahren, dennoch eignen sie 
sich nicht als Monotherapie, bewähren sich jedoch zum Teil als ergänzende Verfahren zur konventionellen Pharmakotherapie oder Psychotherapie, wobei das Risiko möglicher Wechselwirkungen mit herkömmlichen Medikamenten nicht übersehen werden darf (vgl. Freeman et al. 2010).

Für die Forschung ist nicht zuletzt der Befund aus der von Freeman et al. (2010) durchgeführten Metaanalyse interessant, demzufolge die Placebo-Wirkung bei den KM-Verfahren geringer als bei den konventionellen Psychopharmaka war: Dies erklären die Autoren damit, dass Patienten mit einer leichten bis mittelschweren Depression auch vergleichsweise geringere Erwartungen an das Heilmittel haben und bei geringeren Erfolgserwartungen auch ein geringerer Placebo-Effekt eintritt.

Die in Tabelle 18 vorgestellten Reviews und Metaanalysen beziehen sich auf unipolare Depressionen. Bei bipolaren Depressionen ist jedoch von einem teilweise anderen Wirkungsmechanismus auszugehen: So kann z.B. das bei unipolaren Depressionen bewährte Johanniskraut die manische Phase bei bipolaren Depressionen verstärken (Andreescu et al. 2008) und die ebenfalls durchaus bewährten Omega-3-Fettsäuren können in der manischen Phase wirkungslos bleiben (Sarris et al. 2012), und dies vor dem Hintergrund, dass die Studienlage zur Wirkung von KM-Methoden bei Manien ohnehin unzureichend ist (Sarris et al. 2011).

Insgesamt kann festgestellt werden, dass sich einige KM-Methoden den üblicherweise verordneten Antidepressiva bei leichter bis mittelschwerer Depression als gleichwertig, wenn nicht gar als überlegen erweisen. Unter den KMMethoden werden als besonders wirksam betrachtet: am deutlichsten Johanniskraut bei leichten bis mittelschweren Depressionen, etwas weniger Folate, Omega-3-Fettsäuren und S-Adenosylmethionine (SAMe) (vgl. Mischoulon 2007, Fava 2010, Nahas \& Sheikh 2011). Ähnlich resümieren Qureshi \& Al-Bedah (2013) auf der Basis einer Literaturanalyse, dass sich bei Depresionen insbesondere Lichttherapie, Johanniskraut Rosenwurz, Omega-3-Fettsäuren, Yoga, Akupunktur, Aufmerksamkeitsmeditation, Bewegung, Schlafentzug und SAMe bewährt haben; zu Ayurveda und Homöopathie würden zu wenige Studien für eine Beurteilung der Bewährung vorliegen. Insgesamt hätten sich diese Mittel und Methoden vor allem bei leichten bis mittleren (nicht schweren) Depressionen bewährt und könnten in leichteren Fällen auch der Einnahme von Psychopharmaka vorgezogen werden; dies gelte auch für die sog. Wochenbett-Depression oder für Fälle, in denen durch die Einnahme von Antipsychotika Leberschädigungen zu erwarten sind. Tabelle 18 gibt noch einmal einen Überblick über Einzelbefunde.

Tab. 18 Bewährung von KM-Methoden bei Depressionsstörungen

\begin{tabular}{|c|c|c|}
\hline Methode & Ergebnis in Kurzform & Quelle \\
\hline $\begin{array}{l}\text { Methodenver- } \\
\text { gleich }\end{array}$ & $\begin{array}{l}\text { hohe Evidenz für Schlafentzug und Free and Easy Wanderer Plus } \\
\text { etwas weniger für Gymnastik, Yoga, Lichttherapie, Omega-3- } \\
\text { Fettsäuren, SAMe und Tryptophan }\end{array}$ & $\begin{array}{l}\text { Ravindran \& da Silva } \\
2013\end{array}$ \\
\hline
\end{tabular}




\begin{tabular}{|c|c|c|}
\hline Methode & Ergebnis in Kurzform & Quelle \\
\hline $\begin{array}{l}\text { Methodenver- } \\
\text { gleich }\end{array}$ & $\begin{array}{l}\text { hohe Evidenz für Johanniskraut } \\
\text { etwas weniger für SAMe und Bewegung/Gymnastik } \\
\text { geringe Evidenz für Folate und Omega-3-Fettsäuren } \\
\text { keine Evidenz für Akupunktur }\end{array}$ & Nahas \& Sheikh 2011 \\
\hline $\begin{array}{l}\text { Methodenver- } \\
\text { gleich }\end{array}$ & $\begin{array}{l}\text { hohe Evidenz für Lichttherapie bei saisonaler Depression } \\
\text { etwas weniger für Gymnastik, Yoga und Schlafentzug, Omega-3- } \\
\text { Fettsäuren und SAMe }\end{array}$ & Ravindran et al. 2009 \\
\hline $\begin{array}{l}\text { Methodenver- } \\
\text { gleich }\end{array}$ & $\begin{array}{l}\text { hohe Evidenz für Johanniskraut } \\
\text { etwas weniger für SAMe } \\
\text { keine Evidenz für Lecithin }\end{array}$ & Morgan \& Jorm 2008 \\
\hline $\begin{array}{l}\text { Methodenver- } \\
\text { gleich }\end{array}$ & $\begin{array}{l}\text { hohe Evidenz für Johanniskraut } \\
\text { etwas weniger für SAMe, Aerobic und Entspannungstechniken, } \\
\text { Lichttherapie (bei Winterdepression) und Schlafentzug } \\
\text { keine Evidenz für Qigong, Tai Chi und Aromatherapie }\end{array}$ & Ernst 2007 \\
\hline $\begin{array}{l}\text { Methodenver- } \\
\text { gleich }\end{array}$ & $\begin{array}{l}\text { hohe Evidenz für Johanniskraut } \\
\text { etwas weniger für 5-Hydroxy-Tryptofan, Aerobic und Akupunktur } \\
\text { keine Evidenz für Aromatherapie }\end{array}$ & Tachil et al. 2007 \\
\hline \multirow[t]{3}{*}{ Phytopharmaka } & hohe Evidenz für Johanniskraut & $\begin{array}{l}\text { Sarris \& Kavanagh } \\
2009\end{array}$ \\
\hline & $\begin{array}{l}\text { andere Kräuter wie Safran, Lavendel, Borretsch oder Rosenwurz } \\
\text { in ihrer Wirkung noch nicht genügend überprüft }\end{array}$ & Dwyer et al. 2010 \\
\hline & $\begin{array}{l}\text { chinesische Kräuter wie Xiao Yao San in ihrer Wirkung noch nicht } \\
\text { genügend überprüft }\end{array}$ & $\begin{array}{l}\text { Butler \& Pilkington } \\
2013 \\
\text { Zhang et al. } 2012\end{array}$ \\
\hline $\begin{array}{l}\text { Bewegungsübun- } \\
\text { gen }\end{array}$ & $\begin{array}{l}\text { hohe Evidenz für hoch-energetische Gymnastik-Übungen und } \\
\text { häufige Aerobic-Übungen pro Woche }\end{array}$ & Saeed et al. 2010 \\
\hline Qigong & $\begin{array}{l}\text { widersprüchliche Ergebnisse } \\
\text { stärkt eher das allgemeine Wohlbefinden }\end{array}$ & $\begin{array}{l}\text { Oh et al. } 2013 \\
\text { Wang et al. } 2013\end{array}$ \\
\hline Akupunktur & vorliegende Ergebnisse wenig zuverlässig & Ernst et al. 2011 \\
\hline Neurofeedback & vielversprechend, aber noch nicht genügend überprüft & $\begin{array}{l}\text { Dias \& van Deusen } \\
2011\end{array}$ \\
\hline Lichttherapie & $\begin{array}{l}\text { bewährt sich bei saisonalen Depressionen, erscheint aber vielver- } \\
\text { sprechend auch bei nicht saisonalen Verstimmungen }\end{array}$ & $\begin{array}{l}\text { Michalsen \& Kessler } \\
2010 \\
\text { Ravindran et al. } 2010\end{array}$ \\
\hline Massage & geringe Evidenz, weniger effektiv als Akupunktur & Coelho et al. 2008 \\
\hline
\end{tabular}


Anzumerken ist, dass nicht alle KM-Verfahren frei sind von irgendwelchen Nebenwirkungen. Dies gilt insbesondere für Johanniskraut, das mit Medikamenten interagieren kann (vgl. Andreescu et al. 2008), sodass gelegentlich auch vom Gebrauch abgeraten wird (Shelton 2009). Im Übrigen erweist sich Johanniskraut nur wirksam bei leichteren bis mittleren Depressionen (Linde et al. 2008), kann aber mit synthetischen Antidepressiva gleichwertig oder jenen sogar überlegen sein (Röder et al. 2004, Linde 2009).

Besonders widersprüchlich ist die Einschätzung der Akupunktur bei Depressionen. Während Zhang et al. (2010) diese sogar als Alternative zu Antidepressiva ansehen, betonen Wang et al. (2008) die Schwierigkeit des Vergleichs bei unterschiedlichen Depressionsstärken; demgegenüber gilt die Evidenz der Akupunktur bei Leo \& Ligot (2007) sowie Smith et al. (2010) als unzureichend. Ernst et al. (2011) stellen in einem Vergleich der bis dahin vorliegenden Reviews fest, dass 5 von 8 Reviews eine positive Einschätzung der Akupunktur bei Depressionen geben, diese jedoch sämtlich chinesischer Herkunft sind.

Schließlich verdienen auch Beobachtungsstudien Beachtung, wie sie etwa im Rahmen der anthroposophischen Medizin durchgeführt wurden und bei denen Methodenkombinationen zur Anwendung kamen (z.B. Hamre et al. 2007). So sinnvoll Methodenkombinationen in der Praxis sein mögen, so erschweren sie doch eine differenzierte Bewertung nach dem Beitrag einer einzelnen Methode oder eines einzelnen methodischen Elements. Dieses Problem zeigt sich auch bei der Aromatherapie (Lee et al. 2011), beim Yoga (Pilkington et al. 2005, Uebelacker et al. 2010) oder bei der Massage (Coelho et al. 2008).

Unter den Phytopharmaka erscheint zwar die Wirkung von Johanniskraut bei leichten und mittleren Depressionen unzweifelhaft, doch kommen auch noch weitere Substanzen wie Safran, Lavendel, Natternkopf oder Rosenwurz mit einer potenziell Angst und Depression mindernden Wirkung in Betracht (vgl. Dwyer et al. 2010). Über die Bewährung von Kava bei generalisierten Angststörungen hatten bereits Sarris \& Kavanagh (2009) berichtet (Kava in Deutschland nicht mehr im Handel). In einer neueren Übersicht zeigt sich außerdem beispielsweise eine anxiolytische Wirkung von (oral verabreichtem) Lavendel (Perry et al. 2012). Potenziell sind diese Heilkräuter auch geeignet für die Anwendung bei PanikStörungen, speziellen Phobien und Zwangsstörungen (Sarris 2007).

Bestimmte Methoden der sog. Mind-Body-Medizin wie Achtsamkeitsmeditation oder Stressreduktionstechniken haben sich bei Ängsten und Depressionen tendenziell bzw. in Kombination mit einer psychologischen Therapie als wirksam erwiesen (Marchand 2012), wobei diese Wirkung in erster Linie auf die Senkung von körperlichen Spannungen und Reduktion von belastenden Gedanken zurückgeführt werden dürfte. Chen et al. (2012) weisen entsprechend darauf hin, dass meditative Praktiken nicht die Angststörung an sich beeinflussen, sondern nur deren Symptome. Demgegenüber meinen Edenfeld \& Saeed (2012), die Wirkung beruhe nicht nur auf Entspannung, sondern auch bestimmte Denkmuster 
und Formen der emotionalen Verarbeitung könnten mit diesen Praktiken verändert werden.

\subsubsection{Schizophrenie}

Schizophrenie ist eine chronische Krankheit, die in der Regel mit Antipsychotika behandelt wird und deren Nebenwirkungen nicht unerheblich sind. Deshalb erhofft man sich von der KM zumindest eine unterstützende Rolle im Hinblick auf die Minderung von Nebenwirkungen. In einem neueren Methodenvergleich von Helgason \& Sarris (2013) scheinen sich vor allem Musiktherapie, Meditation und Achtsamkeitstechniken als nützlich bei der Unterstützung einer pharmakologischen Therapie zu erweisen. In einigen Studien haben sich Yoga, Atem- und Entspannungstechniken bewährt. Für andere Verfahren wie Hypnose, Thermaloder EMG-Biofeedback, Tanz- oder Dramatherapie liegen derzeit noch zu wenige aussagekräftige Daten vor. Daneben wird gegenwärtig die Eignung von Verfahren für die Schizophrenie überprüft, die sich bei anderen Indikationen bewährt haben, so z.B. Ingwer (Gingko biloba), der sich bei Demenz bewährt hat (Brondino et al. 2013).

- Phytotherapie: Insbesondere bestimmte chinesische Heilkräuter scheinen geeignet zu sein, die Nebenwirkungen von Antipsychotika zu mildern (Rathbone et al. 2005), wobei Ginkgo an erster Stelle zu nennen ist (Singh et al. 2010). Zur Minderung von Brechreiz und Übelkeit eignen sich auch die indischen Heilkräuter Brahmyadiyoga und Tagara, die im Rahmen einer ayurvedischen Behandlung Verwendung finden (Agarwal et al. 2007). Als Alternativen zu einer antipsychotischen Behandlung kommen diese Kräuter jedoch nicht infrage.

- Künstlerische Therapien: Bei Musiktherapie mit genügend vielen Sitzungen bessert sich das allgemeine Wohlbefinden (Gold et al. 2005, Halgason \& Sarris 2013). Dies gilt auch für Bewegungstherapie (Gorczynski \& Faulkner 2010). Die Studienlage zur Tanztherapie und zur Kunsttherapie erlaubt derzeit noch keine Aussagen über ihre Bewährung (Xia \& Grant 2009 bzw. Ruddy \& Milnes 2005).

- Sonstige Verfahren: Zur Akupunktur liegen zu wenige und überwiegend chinesische Studien vor, um zu Aussagen über die Bewährung treffen zu können (Lee et al. 2009). Ähnlich stellt sich die Lage bei der Hypnose dar (Izquierdo de Santiago \& Khan 2007). Yoga als ergänzende Therapie wird nach der Übersicht von Vancampfort et al. 2012 als potenziell wirksam, nach der Metananalyse von Cramer et al. (2013) jedoch als wenig geeignet für einen Routineeinsatz bewertet. 


\subsubsection{Demenz}

Obwohl Demenz vom vaskulären Typus oder vom Alzheimer Typus nur selten in Krankenhäusern als Primärdiagnose gestellt, häufig übersehen oder bestenfalls als milde kognitive Beeinträchtigung registriert wird, ist dieses Krankheitsbild eine Herausforderung nicht nur für die Behandlung, sondern auch für die Behandelnden (Ärzte, Pflegekräfte). Demente Patienten fügen sich nicht in den normalen Krankenhausbetrieb, fallen durch Verständnis- und Orientierungsschwierigkeiten auf, halten sich oft nicht an Anweisungen und zeigen oft ein provokantes Verhalten, das sich nachteilig auf die Behandlungsqualität auswirkt. Andererseits müssen sich Krankenhäuser ebenso wie niedergelassene Praxen in den kommenden Jahren aufgrund der demografischen Entwicklung auf eine Zunahme von Patienten mit demenzieller Komorbidität einstellen. Dabei sind die Möglichkeiten der konventionellen Medizin für eine Primärbehandlung oder auch nur für eine Prävention der Alzheimer-Demenz recht eingeschränkt. Neuere Langzeituntersuchungen zeigen, dass die Lebenslage (z.B. Einsamkeit) der betroffenen Patienten, damit möglicherweise verbundene Depressionen sowie das Ausmaß an kognitiver und sozialer Aktivität das Demenzrisiko in entscheidender Weise erhöht und den Degenerationsprozess bestenfalls verzögern, aber nicht verhindern kann (Wilson et al. 2007, 2010). Daneben scheinen Faktoren des Lebensstils (Ernährung, Rauchen u.ä.) und die damit oft verbundene Gewichtszunahme eine negative Entwicklung zumindest $\mathrm{zu}$ begünstigen (Luchsinger \& Gustafson 2009).

$\mathrm{Zu}$ beachten ist, dass demenziell Erkrankte am häufigsten Vitamine, danach folgend weitere Kräutermischungen zu sich nehmen, sofern sie sich ihrer kognitiven Schwächen bewusst sind. Allerdings informieren nur ca. 50\% der Erkrankten ihren behandelnden Arzt darüber, wie eine deutsche Studie zeigte (Landin et al. 2008).

Aus Sicht der Komplementärmedizin (KM) im Krankenhaus bieten sich hier grundsätzlich zwei verschiedene Ansatzpunkte im Umgang mit Demenz an:

- Phytotherapie: Am erfolgreichsten bei der Milderung demenzieller Symptome scheint die Einnahme von ginkgohaltigen Präparaten zu sein (IQWiG 2008). Ginkgo biloba gilt als relativ sicher, obwohl die Evidenz der entsprechenden Studien noch nicht überzeugend ist (Birks et al. 2007), während May et al. (2009) eine im Hinblick auf die leichte Überlegenheit gegenüber pharmazeutischen Interventionen sowie gegenüber Placebo- und Wartebedingungen insgesamt positive Evidenz bescheinigen können. Ob sich weitere Phytopharmaka aus der chinesischen Medizin als wirksam erweisen, bleibt nach Wang (2010) abzuwarten. Vielversprechend erscheinen hier der Pflanzenextrakt Lycopodium serratum (Fu \& Li 2011) und die Pflanzenformel Yi-GanSan bei Alzheimer-Demenz, welche sedierend wirkt und Agitiertheit mildert (Dos Santos-Neto 2006). 
- Andere Methoden der KM: Relativ ungewiss ist die Evidenz von Akupunktur bei vaskulärer Demenz (Peng et al. 2007). Dies gilt auch für Homöopathie (McCarney et al. 2003), für physische Aktivitäten zur Verminderung von Verhaltensproblemen (Forbes et al. 2008) und für Aromatherapie (Nguyen \& Paton 2008). $\mathrm{Zu}$ den ebenfalls häufig eingesetzten Kunst-, Tanz- und Musiktherapien liegen keine aussagekräftigen Studien vor. Außerdem lassen sie nicht klar erkennen, was das entscheidende Agens (Musik, Bewegung bzw. das soziale Gemeinschaftserleben) ist.

In der Zusammenschau der Versuche von konventioneller Medizin und KM, demenzielle Prozesse zu beeinflussen, kann man feststellen, dass phytotherapeutische und medikamentöse Interventionen sich bisher als wenig erfolgreich erwiesen haben (Kelley \& Knopman 2008). Möglicherweise bietet die - im Alltagsleben von Dementen oft vermisste - soziale Unterstützung einen entscheidenden Ansatzpunkt zumindest für die Besserung des subjektiven Wohlbefindens (Oppikofer et al. 2010), ebenso wie eine auf das Erscheinungsbild der Demenz abgestellte Kommunikation (vgl. Hoefert 2012). Beides scheint in KM-orientierten Krankenhäusern allein schon wegen der verfügbareren Zeit für Patienten eher realisierbar zu sein als in konventionellen Akutkrankenhäusern.

\subsubsection{Zwangsstörungen}

Studien zur KM bei Zwangsstörungen sind sehr selten bzw. sind zu klein für generalisierende Aussagen (vgl. Sarris et al. 2011). Die wenigen Studien zur Wirkung von Johanniskraut zeigen keinen nennenswerten Effekt (Kobak et al. 2005).

\subsubsection{Posttraumatisches Belastungssyndrom}

In einem aufwändigen Peer-Review-Verfahren gelangen Strauss et al. (2011) zu der Feststellung, dass KM-Verfahren nur bedingt in der Lage sind, die Wirkungen einer erfahrenen Traumatisierung (PTSD) zu mildern, wobei Akupunktur das Verfahren mit der höchsten Evidenz ist; meditative Verfahren werden als vielversprechend betrachtet. In einem spezifischen Review zur Akupunktur bei PTSD können Kim et al. (2013) ebenfalls eine hohe, wenn auch nicht zwingende Evidenz bescheinigen.

\subsection{Psychiatrisch-neurologische Indikationen}

\subsubsection{Chronischer Schmerz und Fibromyalgie}

Bei der Fibromyalgie handelt es sich um ein komplexes Phänomen, das durch chronische Schmerzen in unterschiedlichen Körperregionen sowie - nicht zuletzt dadurch bedingten - Schlafstörungen und Erschöpfungszuständen gekenn- 
zeichnet ist. Ähnlich wie bei der klassischen Pharmakotherapie scheint keine KM-Methode allein geeignet zu sein, den unterschiedlichen Erscheinungsformen in gleicher Weise gerecht $\mathrm{zu}$ werden. Deshalb wird gelegentlich bezweifelt, dass die ausschließliche Verwendung von KM - sowohl als Monotherapie oder als Kombinationstherapie - hilfreich ist (de Silva et al. 2010). Vielversprechender dürften hier Kombinationen von körperlichen Methoden mit kognitiver Verhaltenstherapie sein (Casale et al. 2008), zumal eine rein pharmakologische Therapie häufig durch Nebenwirkungen und mangelnde Adhärenz der Patienten belastet ist (Braz et al. 2011). Für vielversprechend wird auch eine Kombination von pharmakologischer Therapie (trizyklische Antidepressiva u.ä.) mit Aerobic und kognitiver Verhaltenstherapie eingeschätzt (Nüesch et al. 2012). Tabelle 19 gibt einen Überblick über Reviews der letzten Jahre zur KM bei chronischem Schmerz und Fibromyalgie.

\section{Tab. 19 Reviews zur KM bei chronischem Schmerz und Fibromyalgie}

\begin{tabular}{|c|c|c|}
\hline Methode & Ergebnis in Kurzform & Quelle \\
\hline Methodenvergleich & $\begin{array}{l}\text { sehr empfehlenswert sind meditative Bewegungsvergleiche (Qigong, } \\
\text { Tai Chi, Yoga) } \\
\text { Akupunktur kann in Betracht gezogen werden } \\
\text { nicht empfehlenswert sind Aufmerksamkeitsbasierte Stressreduktion } \\
\text { und Tanztherapie als Monotherapie } \\
\text { nicht empfehlenswert sind Homöopathie und Nahrungsmittelzusätze }\end{array}$ & $\begin{array}{l}\text { Langhorst et al. } \\
2012\end{array}$ \\
\hline Methodenvergleich & $\begin{array}{l}\text { einige Evidenz zeigen Studien zu Akupunktur, Homöopathie, Hydrothe- } \\
\text { rapie und Massage } \\
\text { keine Evidenz nachweisbar für Chiropraktik }\end{array}$ & $\begin{array}{l}\text { Terry et al. } \\
2012\end{array}$ \\
\hline Methodenvergleich & $\begin{array}{l}\text { empfehlenswert sind generell Mind-Body-Therapien und Balneothera- } \\
\text { pie }\end{array}$ & $\begin{array}{l}\text { Terhorst et al. } \\
2011\end{array}$ \\
\hline Methodenvergleich & $\begin{array}{l}\text { vielversprechend sind Akupunktur und einige meditative Methoden } \\
\text { ebenso Magnesium, I-Carnitine und S-Adenosylmethione }\end{array}$ & $\begin{array}{l}\text { Porter et al. } \\
2010\end{array}$ \\
\hline Methodenvergleich & $\begin{array}{l}\text { Achtsamkeitsmeditation zeigt positive Ergebnisse in verschiedenen } \\
\text { Studien } \\
\text { Akupunktur gemischte Ergebnisse } \\
\text { keine Evidenz für Qigong, Biofeedback und Körperwahrnehmungsthe- } \\
\text { rapie }\end{array}$ & $\begin{array}{l}\text { Baranowsky et } \\
\text { al. } 2009\end{array}$ \\
\hline Methodenvergleich & $\begin{array}{l}\text { starke Evidenz für Aerobic und kognitive Verhaltenstherapie } \\
\text { moderate Evidenz für Massage, Muskelanspannungstraining, Aku- } \\
\text { punktur und Balneotherapie } \\
\text { geringe Evidenz für spinale Manipulation, Körperwahrnehmungstrai- } \\
\text { ning, Vitamine, Phytopharmaka und Ernährungsmodifikation }\end{array}$ & $\begin{array}{l}\text { Schneider et al. } \\
2009\end{array}$ \\
\hline
\end{tabular}




\begin{tabular}{|c|c|c|}
\hline Methode & Ergebnis in Kurzform & Quelle \\
\hline Akupunktur & $\begin{array}{l}\text { Effektivität bei Schmerznachweisbar, wenngleich die Differenz zu } \\
\text { Placebo gering ist } \\
\text { vermutlich tragen noch weitere Faktoren zu diesem Effekt bei }\end{array}$ & $\begin{array}{l}\text { Vickers et al. } \\
2012\end{array}$ \\
\hline Akupunktur & $\begin{array}{l}\text { beim Vergleich von Vor- und Nachuntersuchungen zeigen sich unter- } \\
\text { schiedlich starke Effekte für die Parameter Schmerz, Erschöpfung und } \\
\text { Schlafstörungen } \\
\text { tendenziell größte Effekte bei Elektrostimulation und individualisierter } \\
\text { Akupunktur }\end{array}$ & $\begin{array}{l}\text { Langhorst et al. } \\
2010\end{array}$ \\
\hline Akupunktur & kann für die Behandlung von Fibromyalgie nicht empfohlen werden & $\begin{array}{l}\text { Mayhew \& } \\
\text { Ernst } 2007\end{array}$ \\
\hline Akupunktur & $\begin{array}{l}\text { nur geringe Evidenz, dass A. einer Nicht-Behandlung überlegen ist } \\
\text { gemischte Evidenz für Trigger-Point-A. }\end{array}$ & $\begin{array}{l}\text { Itoh \& Kitakoji } \\
2007\end{array}$ \\
\hline Tuina & Wirkung auch in Kombination mit Moxibustion fraglich & $\begin{array}{l}\text { Kong et al. } \\
2012\end{array}$ \\
\hline Phytopharmaka & $\begin{array}{l}\text { Überlegenheit von Phytopharmaka gegenüberPlacebo und konventio- } \\
\text { nellen Medikamenten } \\
\text { fraglich ist die Sicherheit bei Langzeitgebrauch }\end{array}$ & $\begin{array}{l}\text { de Souza- } \\
\text { Nascimento et } \\
\text { al. } 2013\end{array}$ \\
\hline Massage & $\begin{array}{l}\text { nur mäßige Bestätigung für einen Effekt von Massage } \\
\text { Empfehlung, die Intensität der M. progredient zu steigern }\end{array}$ & $\begin{array}{l}\text { Kalichman } \\
2010\end{array}$ \\
\hline Massage & $\begin{array}{l}\text { starke Effekte für die analgesierende Wirkung von Massage bei unspe- } \\
\text { zifischen Rückenschmerzen } \\
\text { mittlere Effekte bei Schulter- und Kopfschmerzen } \\
\text { mäßige Effekte bei Nackenschmerzen und Karpal- Tunnel-Syndrom }\end{array}$ & Tsao 2007 \\
\hline Homöopathie & $\begin{array}{l}\text { trotz einiger positiver Studien bleibt der Nutzen von H. bei Fibromyal- } \\
\text { gie fragwürdig }\end{array}$ & $\begin{array}{l}\text { Perry et al. } \\
2010\end{array}$ \\
\hline Chiropraktik & keine Evidenz für die Effektivität von Chiropraktik bei Fibromyalgie & Ernst 2009 \\
\hline Meditation & tendenziell Besserung von Fibromyalgie-relevanten Symptomen & $\begin{array}{l}\text { Kozasa et al. } \\
2012\end{array}$ \\
\hline
\end{tabular}

Bei bestimmten Erkrankungen wie Osteoarthritis, bei denen Schmerz ein typisches Begleitsymptom ist, können KM-Verfahren zu kleinen Verbesserungen der Befindlichkeit beitragen. Die Evidenz ihrer Wirksamkeit ist allerdings nicht sonderlich hoch (vgl. Macfarlane et al. 2012). 


\subsubsection{Restless-Legs-Syndrom}

Das Restless-Legs-Syndrom (RLS) ist gekennzeichnet durch eine Reihe von Missempfindungen wie Kribbeln, Schmerzen, Wärmegefühle und Ziehen in den Beinen und Füßen, die auch im Ruhe- und Entspannungszustand auftreten. Bisherige Versuche, die Symptomatik mit Hilfe von KM-Methoden zu mindern, beschränken sich auf die Akupunktur, wobei die Evidenz für eine Wirksamkeit allerdings unzureichend ist (Cui et al. 2008).

\subsection{Sonstige psychiatrisch und psychosomatisch relevante Störungen}

\subsubsection{Chronisches Erschöpfungssyndrom}

Die Wirkung von KM-Methoden bei chronischem Erschöpfungssyndrom wird skeptisch bis negativ beurteilt. Alraek et al. (2010) fanden in ihrem Review nur eine geringe Evidenz für die Wirksamkeit von KM-Methoden. Auch van Houdenhove et al. (2010) betonen, dass gegenwärtig Nahrungszusätze oder andere KMVerfahren keinen Ersatz für - durchaus wirksame - psychologische Therapien darstellen können. Ähnlich hatten dies bereits Porter et al. (2006) für die Homöopathie festgestellt; lediglich für die Wirkung von Massage, essenziellen Fettsäuren und Magnesium konnten sie eine positive Wirkung erkennen. Auch die Wirkung von Yoga wird als schwach bezeichnet (Böhm et al. 2012). Als potenziell wirksam betrachten Porter et al. (2010) dagegen Akupunktur, Meditation sowie Magnesium, I-Carnitine und S-Adenosylmethionine (SAMe), welche auch ergänzend bei Ängsten und Depressionen verwendet werden. Chinesische Untersuchungen, nach denen vor allem Akupunktur und Moxibustion wirksam sein sollen, haben deutliche methodische Schwächen, die ihre Aussagekraft mindern (Adams et al. 2009).

Die vorliegende Uneindeutigkeit bei der Beurteilung der Wirksamkeit dürfte damit zusammenhängen, dass chronische Erschöpfung sowohl somatische Ursachen haben kann (und damit bestimmte Defizite durch KM-Produkte ausgeglichen werden können), als auch psychische Ursachen, die im Rahmen der KM bestenfalls mit Methoden der Mind-Body-Medizin (Meditation u.ä.) anzugehen sind, jedoch mit Hilfe der kognitiven Verhaltenstherapie am erfolgreichsten behandelt werden können (Chambers et al. 2006).

\subsubsection{Essstörungen}

Der Beitrag der KM zur Bewältigung des gesundheitspolitisch vorrangigen Problems der Essstörungen (Anorexie, Übergewicht, binge eating usw.) ist bisher relativ gering. So werden beispielsweise phytotherapeutisch zwei Formeln der 
Kampo-Medizin für die Reduzierung und Verhinderung von Übergewicht mit noch nicht abschätzbarem Erfolg favorisiert (Yamakawa et al. 2013). Daneben scheint auch Achtsamkeitszentrierte Meditation vielversprechend bei der Bewältigung von Übergewicht und anderen Essstörungen (Sojcher et al. 2012).

\subsubsection{Schlafstörungen}

Nach ihrer Evidenz geordnet scheinen vor allem Akupressur, Tai Chi und Yoga wirksam bei der Reduzierung von Schlafstörungen, während für Akupunktur und L-Tryptophan widersprüchliche Befunde vorliegen und sich Phytopharmaka (darunter Baldrian) offenbar nicht bewährt haben (Sarris \& Byrne 2011), ebenso wenig wie Homöopathie (Cooper \& Relton 2010). Die bisherigen Befunde zur Akupunktur zeigen zwar tendenziell positive Effekte, erlauben aber noch keine verallgemeinernden Aussagen (Ernst et al. 2011, Huang et al. 2009, Yeung et al. 2009). Nach einer neueren Übersicht (Kozasa et al. 2010) sind vor allem MindBody-Methoden (darunter Yoga, Entspannungstechniken, Tai Chi und Musik) mäßig erfolgreich; auch normale Gymnastik kann schlaffördernd wirken (Yang et al. 2012).

Am deutlichsten ist die Wirksamkeit bei kognitiver Verhaltenstherapie zumindest in der Initialphase einer Therapie (Harsora \& Kessmann 2009).

\subsubsection{Substanzabhängigkeit}

In der stationären Psychiatrie werden häufig auch Fälle von chronischer Substanzabhängigkeit primär behandelt oder z.B. bei Angst- und Depressionsstörungen mitbehandelt. Hier stellt sich die Frage, inwieweit die Komplementärmedizin (KM) eine Alternative oder zumindest eine Ergänzung bieten kann. Generell muss zu dieser Frage festgestellt werden, dass die bisherigen Anwendungen der KM als nicht sonderlich erfolgreich einzustufen waren (Dean 2006) bzw. dass lediglich der Akupunktur und einigen Phytopharmaka eine gewisse Wirksamkeit bescheinigt wird (Behere et al. 2009). Die wichtigsten Ergebnisse lassen sich wie folgt zusammenfassen:

- Phytotherapie: In den letzten Jahren wurde diskutiert, inwieweit bestimmt Heilkräuter den Alkoholkonsum dämpfen können (Dean 2006, Abenavoli et al. 2009). Förderlich für die Abstinenz scheint das japanische Kudzu (Pueraria lobata) zu sein, während zum chinesischen Danshen (Salvia milthiorrhiza) lediglich Erfahrungen aus Tierstudien vorliegen und auch für Jonanniskraut (Hypericum perforatum) nur vorläufige, tendenziell positive Werte berichtet werden. Bei Entzugserscheinungen (Alkohol, Nikotin und andere Drogen) zeigt sich Johanniskraut insoweit erfolgreich, als es Unruhe, Angst und Schlaflosigkeit mindern hilft. Einen ähnlichen Effekt hat Baldrian (Valeriana officinalis). Als potenziell erfolgreich beim Opiatentzug gelten Passionsblume 
(Passiflora incarnata), Rosmarin (Rosmarinus officinalis) und Mohn (Papaver rhoeas). Ob ein Entzug von Heroin mit Hilfe chinesischer Kräuter gelingt, gilt bisher als unklar (Liu et al. 2009). Alkoholische Nachwirkungen wie Übelkeit oder Mundtrockenheit lassen sich offenbar mit Feigenkaktus (Opuntia ficus indicia) mindern, während Artischocke (Cynara scolymus) wenig erfolgreich zu sein scheint. Inwieweit sich alkoholbedingte Leberschädigungen durch Mariendistel (Silybum Marianum) vermindern lassen, ist noch umgewiss. Wenig wirksam scheint hier Vitamin E, Liv 52 oder Polyenylphosphatidylcholin zu sein. Insgesamt ist die Studienlage dadurch gekennzeichnet, dass entweder nur Tierstudien oder $\mathrm{zu}$ kleine Studien vorliegen, um daraus verallgemeinerbare Aussagen abzuleiten.

- Andere Methoden: Im Bereich der Substanzabhängigkeit haben sich Akupunktur ebenso wie Akupressur, Lasertherapie oder Elektrostimulation (für Nikotinentwöhnung) bisher nicht bewährt; dies gilt auch für Akupressur bei der Raucherentwöhnung (White et al. 2007). EEG- Biofeedback in Kombination mit herkömmlichen Therapien scheint dagegen tendenziell erfolgversprechend zu sein (Sokhadze et al. 2008).

\subsection{Bewährung einzelner KM-Methoden}

In recht allgemeiner Form werden die typischen Methoden der KM bei Kemper (2007) und - mit überwiegend positiven Bewertungen - bei Williams et al. (2012) vorgestellt. Eine Einschätzung der jeweiligen Wirksamkeit dieser Methoden kann den obigen, indikationsbezogenen Darstellungen entnommen werden. Auf die methodologischen Probleme der entsprechenden Studien hatten bereits Ernst et al. (2006) sowie Ravindran et al. (2009) grundsätzlich hingewiesen. Sie bestehen im Wesentlichen in der Studiengröße, im tatsächlichen Ausschluss von PlaceboEffekten und in ethischen Problemen bei der Einrichtung von WartelistenKontrollgruppen bei anstehenden Gesundheitsproblemen (z.B. bei Depressionen, die unbehandelt das Suizidrisiko erhöhen könnten). Dennoch lassen sich für die Psychiatrie einige verallgemeinernde Aussagen zur Bewährung von KM-Methoden formulieren, die in der Tabelle 20 zusammengefasst werden.

Tab. 20 Zusammenfassende Bewertung einzelner KM-Methoden

\begin{tabular}{|c|c|c|}
\hline Methode & Bewertung & Quelle \\
\hline \multirow[t]{5}{*}{ Phytopharmaka } & \multirow[t]{4}{*}{ Johanniskraut bei Depressionen bewährt } & Larzelere et al. \\
\hline & & 2010 \\
\hline & & Linde 2009 \\
\hline & & Ernst 2006 \\
\hline & $\begin{array}{l}\text { Rosenwurz und Safran bei Depressionen untersuchungs- } \\
\text { würdig }\end{array}$ & Sarris 2007 \\
\hline
\end{tabular}




\begin{tabular}{|c|c|c|}
\hline Methode & Bewertung & Quelle \\
\hline & $\begin{array}{l}\text { Passionsblume, Minze, chinesische Jujube bei Angst } \\
\text { untersuchungswürdig } \\
\text { Kava bei Panikstörungen und Zwangsstörungen untersu- } \\
\text { chungswürdig }\end{array}$ & \\
\hline & $\begin{array}{l}\text { Kava bei Angststörungen bewährt, Inositol und Baldrian } \\
\text { vielversprechend } \\
\text { Inositol, Folate und Safran bei Depressionen unzu- } \\
\text { reichende Evidenz }\end{array}$ & $\begin{array}{l}\text { Larzalere et al. } \\
2010\end{array}$ \\
\hline & $\begin{array}{l}\text { Ginkgo bei Demenz, aber nicht bei Schizophrenie be- } \\
\text { währt }\end{array}$ & $\begin{array}{l}\text { Brondino et al. } \\
2013\end{array}$ \\
\hline Nahrungsergänzungsmittel & $\begin{array}{l}\text { Omega-3-Fettsäuren und L-Tryptophane bei Depressionen } \\
\text { bewährt }\end{array}$ & $\begin{array}{l}\text { Lakhan \& Vieira } \\
2008\end{array}$ \\
\hline Körperliche Verfahren & Gymnastik und Massage bei Depressionen bewährt & $\begin{array}{l}\text { Ernst } 2006 \\
\text { Kemper \& } \\
\text { Shannon } 2007\end{array}$ \\
\hline Entspannungsverfahren & $\begin{array}{l}\text { Entspannungsverfahren bei Depressionen und Angststö- } \\
\text { rungen bewährt }\end{array}$ & Ernst 2006 \\
\hline Akupressur & $\begin{array}{l}\text { zur Schmerzreduzierung geeignet } \\
\text { geeignet bei Schlafstörungen, Übelkeit und Schmerz }\end{array}$ & $\begin{array}{l}\text { Chen \& Wang } \\
2013 \\
\text { Robinson et al. } \\
2011\end{array}$ \\
\hline Akupunktur & $\begin{array}{l}\text { geeignet bei Depression und Angst } \\
\text { widersprüchlich bei Substanzmissbrauch }\end{array}$ & $\begin{array}{l}\text { Samuels et al. } \\
2008\end{array}$ \\
\hline Shiatsu & insgesamt geringe Evidenz & $\begin{array}{l}\text { Robinson et al. } \\
2011\end{array}$ \\
\hline Tai Chi & $\begin{array}{l}\text { steigert das allgemeine Wohlbefinden } \\
\text { keine indikationsspezifische Wirksamkeit }\end{array}$ & $\begin{array}{l}\text { Wang et al. } \\
2010\end{array}$ \\
\hline Yoga, Chigong & dgl. & $\begin{array}{l}\text { Posadzki et al. } \\
2010\end{array}$ \\
\hline Achtsamkeitsmeditation & $\begin{array}{l}\text { dgl., aber auch spezifische Wirksamkeit bei Ängsten } \\
\text { und Depressionen }\end{array}$ & $\begin{array}{l}\text { Marchand 2012, } \\
2013 \\
\text { Edenfeld \& } \\
\text { Saeed } 2012\end{array}$ \\
\hline Homöopathie & $\begin{array}{l}\text { geeignet für Fibromyalgie und chronisches Erschöp- } \\
\text { fungssyndrom, weniger für Angst- und Stresssymptome }\end{array}$ & $\begin{array}{l}\text { Davidson et al. } \\
2011\end{array}$ \\
\hline
\end{tabular}


Es wird deutlich, dass die KM einerseits - wie auch die konventionelle medizinische Psychiatrie - einem „materiellen“ Paradigma folgt, nach dem sich psychische Störungen auf irgendwelche Defizite von „Stoffen“ bzw. auf organische Fehlfunktionen zurückführen lassen. Andererseits - und dies unterscheidet sie von der konventionellen Psychiatrie - vertraut sie auf „spirituelle“ Prozesse, für die nur schwer irgendwelche materiellen Substrate zu finden sind bzw. ein materieller Wirkungsnachweis $\mathrm{zu}$ finden ist. Die ungenügende Beachtung von Komorbiditäten (z.B. zwischen Depressionen und rheumatologisch relevanten Symptomen, vgl. Bruti et al. 2012) führt, indem Monotherapien favorisiert und bestenfalls additiv ergänzt werden, $z u$ einem verkürzen Behandlungsansatz, der dann bei Ängsten und Depressionen zu rein symptomatischen Wirkungen führt (van der Watt et al. 2008). Die unzureichende Evidenz mancher KM-Methoden kontrastiert oft mit ihrer Akzeptanz und Popularität bei den Nutzern, was erstens die Notwendigkeit einer besseren Aufklärung bzw. einer „Entmystifizierung“ deutlich werden lässt, zweitens aber auch eine Grundlage für eine bessere Adhärenz bei denjenigen Nutzern darstellt, die entweder synthetische Psychopharmaka wegen ihrer Nebenwirkungen nicht vertragen oder jene aus weltanschaulichen Gründen ablehnen und KM-Methoden deshalb bevorzugen.

\section{Zusammenfassung}

Ängste und Depressionen sind die häufigsten Indikationen der Psychiatrie. Entsprechend konzentrieren sich die meisten Verfahren der Komplementärmedizin auf diese beiden Krankheitsbilder bzw. liegen dazu die meisten und aussagekräftigsten Studien vor. Danach sind einige dieser Methoden geeignet, zumindest die Angstsymptomatik zu reduzieren oder den basalen Gefühlszustand bei leichten bis mittleren Depressionen zu bessern. Die Überprüfung der Wirksamkeit bei weiteren psychiatrischen Syndromen, insbesondere bei solchen, die zu den sog. funktionellen Störungsbildern gezählt werden, bleibt eine Zukunftsaufgabe.

\section{Literatur}

Abenavoli, L., Capasso, F., Addolorato, G. (2009). Phytotherapeutic approach to alcohol dependence: new old way? Phytomedicine, 16(6-7), 638-644.

Adams, D., Wu, T., Yang, X., Tai, S., Vohra, S. (2009). Traditional Chinese medicinal herbs for the treatment of idiopathic chronic fatigue und chronic fatigue syndrome. Cochrane Database Syst Rev, 4, CD006348.

Agarwal, V., Abhijnhan, A., Raviraj, P. (2007). Ayurvedic medicine for schizophrenia. Cochrane Rev, 4, CD006867.

Alderman, C.P. (2003). Complementary medicine use by psychiatric patients of an Australian hospital. Ann Pharmacother, 37(12), 1779-1784.

Alraek, T., Lee, M.S., Choi, T.-Y., Cao, H., Liu, J. (2011). Complementary and alternative medicine for patients with chronic fatique syndrome: A systematic review. BMC Complement Altern Med, 11:87.

Andreescu, C., Mulsant, B.H., Emanuel, J.E. (2008). Complementary and alternative medicine in the treatment of bipolar disorder - a review of the evidence. I Affect Disord, 110(1-2), 16-26.

Baranowsky, I., Klose, P., Musial, F., Häuser, W., Dobos, G., Langhorst, I. (2009). Qualitative systematic review of randomized controlled trials on complementary and alternative medicine treatments in fibromyalgia. Rheumatol Int, 30(1), 1-21. 
Behere, R.V., Muralidharan, K., Benegal, V. (2009). Complementary and alternative medicine in the treatment of substance use disorders - a review of the evidence. Drug Alcohol Rev, 28(3), 292-300.

Birks, I., Grimley Evans, I. (2007). Ginkgo biloba for cognitive impairment and dementia. Cochrane Rev, 2, CD003120.

Böhm, K., Ostermann, T., Milazzo, S., Büssing, A. (2012). Effects of yoga interventions on fatigue: a meta-analysis. Evid Based Complement Alternat Med, ID 124703.

Brondino, N., De Silvestri, A., Re, S., Lanati, N., Thiemann, P., Verna, A., Emanuele, E., Politi, P. (2013). A Systematic Review and Meta-Analysis of Ginkgo biloba in Neuropsychiatric Disorders: From Ancient Tradition to Modern-Day Medicine. Evid Based Complement Alternat Med. 2013; 915691.

Bruti, G., Magnotti, M.C., lanetti, G. (2012). Migraine and depression: bidirectional co-morbidities? Neurol Sci, 33, Suppl. 1, S107-S109.

Busch, M.A., Maske, U.E., Ryl, L., Schlack, R., Hapke, U. (2013). Prävalenz von depressiver Symptomatik und diagnostizierter Depression bei Erwachsenen in Deutschland. Bundesgesundhbl, 56, 733-739.

Butler, L., Pilkington, K. (2013). Chinese herbal medicine and depression: the research evidence. Evid Based Complement Alternat Med, doi: 1155/2013/739716.

Bystritsky, A., Hovav, S., Sherbourne, C., Stein, M.B., Rose, R.D., Campbell-Sills, L., Golinelli, D., Sullivan, G., Craske, M.G., Roy-Byrne, P.P. (2012). Use of complementary and alternative medicine in a large sample of anxiety patients. Psychosomatics, 53(3), 266-272.

Casale, R., Cazzola, M., Arioli, G., Gracely, R.H., Ceccherelli, F., Atzeni, F., Stisi, S., Cassisi, G., Altomonte, L., Alciati, A., Leardini, G., Gorla, R., Marsico, A., Torta, R., Giamberardino, M.A., Buskila, D., Spath, M., Marinangeli, F., Bazzichi, L., Di Franco, M., Biasi, G., Salaffi, F., Carignola, R., Sarzi-Puttini, P., Italian Fibromyalgia Network (2008). Non pharmacological treatments in fibromyalgia. Reumatismo, 60, Suppl. 1, 59-69.

Chambers, D., Bagnall, A.-M., Hempel, S., Forbes, C. (2006). Interventions for the treatment, management and rehabilitation of patients with chronic fatigue syndrome/myalgic encephalomyelitis: an updated systematic review. J Royal Soc Med, 99, 506-520.

Chen, K.W., Berger, C.C., Manheimer, E., Forde, D., Magidson, J., Dachman, L., Lejuez, C.W. (2012). Meditative therapies for reducing anxiety: a systematic review and meta-analysis of randomized controlled trials. Depress Anxiety, 29(7), 545-562.

Chen, Y.W., Wang, H.H. (2013). The effectiveness of acupressure on relieving pain: A systematic review. Pain Manag Nurs, doi: 10.1016/j.pmn.2012.005.

Coelho, H.F., Boddy, K., Ernst, E. (2008). Massage therapy for the treatment of depression: a systematic review. Int I Clin Pract, 62(2), 325-333.

Cooper, K.L., Relton, C. (2010). Homeopathy for insomnia: a systematic review of research evidence. Sleep Med Rev, 14(5), 329-337.

Cui, Y., Wang, Y., Liu, Z. (2008). Acupuncture for restless legs syndrome. Cochrane Rev, CD006457.

Davidson, J.R., Crawford, C., Ives, J.A., Jonas, W.B. (2011). Homeopathic treatments in psychiatry: a systematic review of randomized placebo-controlled studies. J Clin Psychiatry, 72(6), 795-805.

de Silva, V., El-Metwally, A., Ernst, E., Lewith, G., Macfarlane, G.J. (2010). Evidence for the efficacy of complementary and alternative medicines in the management of fibromyalgia: a systematic review. Rheumatology, 49, 1063-1069.

de Souza Braz, A., de Paula, A.P., Melo Diniz, M.F., de Almeida, R.N. (2011). Non-pharmacological therapy and complementary and alternative medicine in fibromyalgia. Rev Bras Reumatol, 51(3), 269-282.

de Souza Nascimento, S., Desantana, I.M., Nampo, F.K., Ribeiro, E.A., da Silva, D.L., Araujo-Junior, J.X., da Silva Almeida, J.R., Bonjardim, L.R., de Souza Araujo, A.A., Quintans-Junior, L.J. (2013). Efficacy and safety of medical plants or related natural products for fibromyalgia: a systematic review. Evid Based Complement Alternat Med, ID 149468.

Dias, A.M., van Deusen, A. (2011). A new neurofeedback protocol for depression. Span I Psychol, 14(1), 374-384.

Dean, A. (2005). Natural and complementary therapies for substance use disorders. Curr Opin Psychiatry, 18, 271276.

Dos Santos-Neo, L.L., de Vilhena Toledo, M.A., Mdeiros-Souza, P., de Souza, P. (2006). The use of herbal medicine in Alzheimer's disease - a systematic review. Evid Based Complement Alternat Med, 3(4), 441-445.

D'Silva, S., Poscablo, C., Habousha, R., Kogan, M., Kligler, B. (2012). Mind-body medicine therapies for a range of depression severity: a systematic review. Psychosomatics, 53(5), 407-423.

Dwyer, A.V., Whitten, D.L., Hawrelak, J.A. (2010). Herbal medicines, other than St. John's Wort, in the treatment of depression: A systematic review. Altern Med Rev, 16(1), 40-49. 
Edenfeld, T.M., Saeed, S.A. (2012). An update on mindfulness meditation as a self-help treatment for anxiety and depression. Psychol Res Behav Manage, 5, 132-141.

Ernst, E. (2006a). Complementary medicine in psychiatry. Die Psychiatrie, 3, 157-165.

Ernst, E. (2006b). Herbal remedies for anxiety - a systematic review of controlled clinical trials. Phytomedicine, 13(3), 205-208.

Ernst, E. (2007). Herbal remedies for depression and anxiety. Adv Psychiatr Treatment, 13, 312-316.

Ernst, E. (2009). Chiropractic treatment for fibromyalgia: a systematic review. Clin Rheumatol, 28(10), 1175-1178.

Ernst, E., Lee, M.S., Choi, T.-Y. (2011). Acupuncture for insomnia? An overview of systematic reviews. Eur I Gen Pract, 17, 116-123.

Ernst, E., Lee, M.S., Choi, T.Y. (2011). Acupuncture for depression? A systematic review of systematic reviews. Eval Health Prof, 34(4), 403-412.

Fava, M. (2010). Using complementary and alternative medicines for depression. I Clin Psychiatry, 71(9), e24.

Forbes, D., Forbes, S., Morgan, D.G., Markle-Reid, M., Wood, I., Culum, I. (2008). Physical activity programs for persons with dementia. Cochrane Rev, 3, CD 006489.

Freeman, M.P., Mischoulon, D., Tedeschini, E., Goodness, T., Cohen, L.S., Fava, M., Papakostas, G.I. (2010). Complementary and alternative medicine in major depressive disorder: A meta-analysis of patient characteristics, placebo-response rates, and treatment outcomes relative to standard antidepressants. I Clin Psychiatry, $71(6), 682-688$.

Fu, L.-M., Li, I.T. (2011). A systematic review of single Chinese herbs for Alzheimer's disease treatment. Evid Based Complement Alternat Med, ID640284.

Gaebel, W. (2012). Psychische Erkrankungen - Ursachen, Prävalenz und Auswirkungen auf die Arbeitsfähigkeit. Arbeitsmed Sozialmed Umweltmed, 47(12), 163-165.

Gold, C., Heldal, T.O., Dahle, T., Wigram, T. (2005). Music therapy for schizophrenia or schizophrenia-like illnesses. Cochrane Rev, 2, CD004025.

Gorczynski, P., Faulkner, G. (2010). Exercise therapy for schizophrenia. Cochrane Rev, 5, CD004412.

Hammond, D.C. (2010). Hypnosis in the treatment of anxiety- and stress-related disorders. Expert Rev Neurother, $10(2), 263-273$.

Hamre, H.J., Witt, C.M., Glöckmann, A., Ziegler, R., Willich, S.N., Kiene, H. (2007). Anthroposophische Therapie bei chronischer Depression: eine vierjährige prospektive Kohortenstudie. Der Merkurstab, 3, 208-220.

Hapke, U., Maske, U.E., Scheidt-Nave, C., Bode, L., Schlack, R., Busch, M.A. (2013). Chronischer Stress bei Erwachsenen in Deutschland. Bundesgesundheitsbl, 56, 749-754.

Harfst, T, Marstedt, G. (2009). Psychische Gesundheit in Deutschland: Erkrankungen bleiben oft unentdeckt. Gesundheitsmonitor, 1, 1-7.

Harsora, P., Kessmann, I., (2009). Nonpharmacologic managment of chronic insomnia. Am Fam Phys, 79(2), 125130.

Helgason, C., Sarris, I. (2013). Mind-Body Medicine for schizophrenia and psychotic disorders. Clin Schizophrenia Relat Disord, 7(3), 138-148.

Hoefert, H.-W. (2012). Ärztlicher Umgang mit dementen Patienten im Krankenhaus. In: Hellmann, W. (Hrsg.). Handbuch Integrierte Versorgung. 36. Akt., Juli 2012, 1-11.

Huang, W., Kutner, N., Bliwise, D.L. (2009). A systematic review oft he effects of acupuncture in treating insomnia. Sleep Med Rev, 13(1), 73-104.

Huber, R., Koch, D., Beiser, I., Zschocke, I., Lüdtke, R. (2004). Experience and attitudes towards CAM - a survey of internal and psychosomatic patients in a German university hospital. Altern Ther Health Med, 10(1), 32-36.

Institut für Qualität und Wirtschaftlichkeit im Gesundheitswesen (IQWiG) (2008). Ginkgohaltige Präparate bei Alzheimer Demenz. Abschlussbericht.

Itoh, K., Kitakoji, H. (2007). Acupuncture for chronic pain in Japan: a review. Evid Based Complement Alternat Med, 4(4), 431-434.

Izquierdo de Santiago, A., Khan, M. (2007). Hypnosis for schizophrenia. Cochrane Rev, 4, CD004160.

Jacobi, F., Harfst, T. (2007). Psychische Erkrankungen - Erscheinungsformen, Häufigkeit und gesundheitspolitische Bedeutung. Die Krankenversicherung, 5, 3-6.

Jorm, A.F., Christensen, H., Griffiths, K.M., Parslow, R.A., Rodgers, B., Blewitt, K.A. (2004). Effectiveness of complementary and self-help treatments for anxiety disorders. MJA, 181(7), S29-S46.

Jorm, A.F., Allen, N.B., O'Donnell, C.P., Parslow, R.A., Purcell, R., Morgan, A.J. (2006). Effectiveness of complementary and self-help treatments for depression in children and adolescents. MJA, 185(7), 368-372. 
Jorm, A.F., Morgan, A.J., Hetrick, S.E. (2008). Relaxation for depression. Cochrane Rev, 4, CD007142.

Kalichman, L. (2010). Massage therapy for fibromyalgia symptoms. Rheumatol Int, 30(9), 1151-1157.

Kelley, B.J., Knopman, D.S. (2008). Alternative medicine and Alzheimer's disease. Neurologist, 14(5), 299-306.

Kemper, K.J., Shannon, S. (2007). CAM Therapies to promote healthy moods. Pediatr Clin North Am, 54(6), 901-X.

Kessler, R.C., Soukup, I., Davis, R.B., Foster, D.F., Wilkey, S.A., van Rompay, M.I., Eisenberg, D.M. (2001). The use of complementary and alternative therapies to treat anxiety and depression in the United States. Am I Psychiatry, 158, 289-294.

Kim, Y.D., Heo, I., Shin, B.C., Crwaford, C., Kang, H.W., Lim, J.H. (2013). Acupuncture for posttraumatic stress disorder: a systematic review of randomized controlled trials and prospective clinical trials. Evid Based Complement Alternat Med, doi: 10.1155/2013/615857.

Kirkwood, G., Rampes, H., Tuffrey, V., Richardson, I., Pilkington, K. (2005). Yoga for anxiety: a systematic review of the research evidence. Br / Sports Med, 39, 884-891.

Kobak, K.A., Taylor, L.V., Bystritsky, A., Kohlenberg, C.I., Greist, J.H., Tucker, P., Warner, G., Futterer, R., Vapnik, T. (2005). St John's wort versus placebo in obsessive-compulsive disorder: results from a double-blind study. Int Clin Psychopharmacol, 20(6), 299-304.

Kong, L.J., Fang, M., Zhan, H.S., Yuan, W.A., Pu, J.H., Cheng, Y.W., Chen, B. (2012). Tuina-focussed integrative Chinese medical therapies for inpatients with low back pain: a systematic review and meta-analysis. Evid Based Complement Alternat Med, ID 578305.

Kozasa, E.H., Hachul, H., Monson, C., Pinto jr., L., Csermak Garcia, M., Mello, L.E., Tufik, S. (2010). Mind-body interventions for the treatment of insomnia: a review. Revista Bras Psiquiatrica, 32(4), 437-443.

Kozasa, E.H., Tanaka, L.H., Monson, C., Little, S., Leao, F.C., Peres, M.P. (2012). The effects of meditation-based interventions on the treatment of fibromyalgia. Curr Pain Headache Rep, 16(5), 383-387.

Krisanaprakonkit, T., Sriraj, W., Piyavhatkul, N., Laopaiboon, M. (2006). Meditation therapy for anxiety disorders. Cochrane Rev, 1, CD004998.

Lademann, I., Mertesacker, H., Bebhardt, B. (2006). Psychische Erkrankungen im Fokus der Gesundheitsreporte der Krankenkassen. Psychotherapeutenjournal, 2, 123-129.

Lakhan, S.E., Vieira, K.F. (2008). Nutritional therapies for mental disorders. Nutr I, 7:2.

Landin, I., Frölich, L., Schwarz, S. (2008). Use of alternative therapies in patients with dementia and mild cognitive impairment: a prospective, controlled study. Int / Geriatr Psychiatry, 23(11), 1163-1165.

Langhorst, I., Klose, P., Musial, F., Irnich, D., Häuser, W. (2010). Efficacy of acupuncture in fibromyalgia syndrome - a systematic review with a meta-analysis of controlled clinical trials. Rheumatology, 49, 778-788.

Langhorst, I., Häuser, W., Irnich, D., Speeck, N., Felde, E., Winkelmann, A., Lucius, H., Michalsen, A., Musial, F. (2012). Komplementäre und alternative Verfahren beim Fibromyalgiesyndrom. Schmerz, 26(3), 311-317.

Larzere, M.M., Campbell, I.S., Robertson, M. (2010). Complementary and alternative medicine usage for behavioral health indications. Prim Care, 37(2), 213-236.

Lee, M.S., Shin, B.C., Ronan, P., Ernst, E. (2009). Acupuncture for schizophrenia: a systematic review and metaanalysis. Int I Clin Pract, 63(11), 1622-1633.

Lee, Y.L., Wu, Y., Tsang, H.W., Leung, A.Y., Cheung, W.M. (2011). A systematic review on the anxiolytic effects of aromatherapy in people with anxiety symptoms. I Altern Complement Med, 17(2), 101-108.

Leo, R.J., Ligot, J.S.A. (2007). A systematic review of randomized controlled trials of acupuncture in the treatment of depression. I Affect Disord, 97(1-3), 13-22.

Lin, P.Y., Su, K.P. (2007). A meta-analytic review of double-blind, placebo-controlled trails of antidepressant efficacy of Omega-3 fatty acids. I Clin Psychiatry, 68(7), 1056-1061.

Linde, K. (2009). St. John's wort - an overview. Forsch Komplementmed, 16(3), 146-155.

Linde, K., Berner, M.M., Kriston, L. (2008). St John's wort for major depression. Cochrane Rev, 4, CD000448.

Liu, T.T., Shi, I., Epstein, D.H., Bao, Y.P., Lu, L. (2009). A meta-analysis of Chinese herbal medicine in treatment of managed withdrawel from heroin. Cell Molecul Neurobiol, 29(1), 17-25.

Luchsinger, J.A., Gustafson, D.R. (2009). Adiposity and Alzheimer's disease. Curr Opin Clin Nutr Metab Care, 12(1), 15-21.

Macfarlane, G.J., Paudyal, P., Doherty, M., Ernst, E., Lewith, G., MacPherson, H., Sim, I., Jones, G.T. (2012). A systematic review of evidence fort he effectiveness of prctioner-based complementary and alternative therapies in the management of rheumatic diseases: osteoarthritis. Rheumatology, 51(12), 2224-2233.

Marchand, W.R. (2012). Mindfulness-based stress reduction, mindfulness-based cognitive therapy, and Zen meditation for depression, anxiety, pain, and psychological distress. I Psychiatr Pract, 18(4), 233-252. 
Marchand, W.R. (2013). Mindfulness meditation practices as adjunctive treatments for psychiatric disorders. Psychiatr Clin North Am, 36(1), 141-152.

Martinsen, E.W. (2008). Physical activity in the prevention and treatment of anxiety and depression. Nord I Psychiatry, 62(Suppl. 47), 25-29.

May, B.H., Lit, M., Xue, C.C., Yang, A.W., Zhang, A.L., Owens, M.D., Head, R., Cobiac, L., Li, C.G., Hugel, H., Story, D.F. (2009). Herbal medicine for dementia: a systematic review. Phytother Res, 23(4), 447-459.

Mayhew, E., Ernst, E. (2007). Acupuncture for fibromyalgia - a systematic review of randomized clinical trials. Rheumatology, 46(5), 801-804.

McKarney, R.W., Warner, I., Fisher, P., van Haselen, R. (2003). Homeopathy for dementia. Cochrane Rev, 1, CD003803.

Mead, G.E., Morley, W., Campbell, P., Greig, C.A., McMurdo, M., Lawlor, D.A. (2009). Exercise for depression. Cochrane Rev, 3, CD004366.

Meeks, T.W., Wetherell, J.L., Irwin, M.R., Redwine, L.S., Jeste, D.V. (2007). Complementary and alternative treatments for late-life depression, anxiety, and sleep disturbance: a review of randomized controlled trials. I Clin Psychiatry, 68(10), 1461-1471.

Michalsen, A., Kessler, C. (2010). Multimodale Therapie depressiver Störungen mit Naturheilkunde und Komplementärmedizin. ZKM, 4, 1-6.

Morgan, A.J., Jorm, A.F. (2008). Self-help interventions for depressive disorders and depressive symptoms: a systematic review. Ann Gen Psychiatry, 7:13.

Mukaino, Y., Park, I., White, A., Ernst, E. (2005). The effectiveness of acupuncture for depression - a systematic review of randomized controlled trials. Acupuncture Med, 23(2), 70-76.

Nahas, R., Sheikh, 0. (2011). Complementary and alternative medicine for the treatment of major depression disorder. Can Fam Phys, 57, 659-663.

National Health Service/Scottish Intercollegiate Network (NHS) (2010). Non-pharmaceutical management of depression in adults. A national clinical guideline. Edinburgh.

Nguyen, Q.A., Paton, C. (2008). The use of aromatherapy to treat behavioural problems in dementia. Int I Geriatr Psychiatry, 23(4), 337-348.

Nüesch, E., Häuser, W., Bernardy, K., Barth, J., Jüni, P. (2012). Comparative efficacy of pharmacological and nonpharmacological interventions in fibromyalgia syndrome: network meta-analysis. Ann Rheum Dis, 72(6), 955-962.

Oh, B., Choi, S.M., Inamori, A., Rosenthal, D., Yeung, A. (2013). Effects of qigong on depression: a systematic review. Evid Based Complement Alternat Med, doi: 10.1155/2013/134737.

Oppikofer, S., Albrecht, K., Martin, M. (2010). Auswirkungen erhöhter sozialer Unterstützung auf das Wohlbefinden kognitiv beeinträchtigter älterer Menschen. Z Gerontol Geriatr, 43, 310-316.

Peng, W.N., Zhao, H., Wang, S. (2007). Acupuncture for vascular dementia. Cochrane Rev, 2, CD004987.

Perry, R., Terry, R., Ernst, E. (2010). A systematic review of homoeopathy for the treatment of fibromyalgia. Clin Rheumatol, 29(5), 457-464.

Perry, R., Terry, R., Watson, L.K., Ernst, E. (2012). Is lavender an anxiolytic drug? A systematic review of randomized clinical trials. Phytomedicine, 19(8-9), 825-835.

Pilkington, K., Kirkwood, G., Rampes, H., Fisher, P., Richardson, I. (2005). Homeopathy for depression: a systemat ic review of the research. Homeopathy, 94(3), 153-163.

Pilkington, K., Kirkwoood, G., Rampes, H., Richardson, J. (2005). Yoga for depression: the research evidence. I Affect Disord, 89(1-3), 13-24.

Pilkington, K., Kirkwood, G., Rampes, H., Fisher, P., Richardson, J. (2006). Homeopathy for anxiety and anxiety disorders: a systematic review of the research. Homeopathy, 95(3), 151-162.

Pilkington, K., Kirkwood, G, Rampes, H., Cummings, M., Richardson, J. (2007). Acupuncture for anxiety and anxiety disorders. Acupuncture Med, 25(1-2), 1-10.

Pittler, M.H., Ernst, E. (2003). Kava extract versus placebo for treating anxiety. Cochrane Rev, 1, CD003383.

Porter, N.S., Jason, L.A., Boulton, A., Bothne, N., Coleman, B. (2010). Alternative medical interventions used in the treatment and management of myalgic encephalomyelitis/chronic fatigue syndrome and fibromyalgia. I Altern Complement Med, 16(3), 235-249.

Posadzki, P., Parekh, S., Glass, N. (2010). Yoga and qigong in the psychological prevention of mental health disorders: a conceptual synthesis. Chin J Integr Med, 16(1), 80-86.

Qureshi, N.A., Al-Bedah, A.M. (2013). Mood disorders and complementary and alternative medicine: a literature review. Neuropsychiatr Dis, 9, 639-658. 
Rathbone, J., Zhang, L., Zhang, M., Xia, J., Liu, X., Yang, Y. (2005). Chinese herbal medicine for schizophrenia. Cochrane Rev, 4, CD003444.

Ravens-Sieberer, U., Wille, N., Bettge, S., Erhart, M. (2007). Psychische Gesundheit von Kindern und Jugendlichen in Deutschland. Bundesgesundheitsbl, 5/6, 871-878.

Ravindran, A.V., Lam, R.W., Filteau, M.J., Lespérance, F., Kennedy, S.H., Parikh, S.V., Patten, S.B. (2009). Canadian Network for Mood an Anxiety Treatments (CANMAT) Clinical guidelines for the management of major depressive disorder in adults. V. Complementary and alternative medicine treatments. I Affect Disord, 117(Suppl. 1), S54-S64.

Ravindran, A.V., da Silva, T.L. (2013). Complementary and alternative therapies as add-on to pharmacotherapy for mood and anxiety disorders: A systematic review. J Affect Disord, 150(3), 707-719.

Robinson, N., Lorenc, A., Liao, X. (2011). The evidence for Shiatsu: a systematic review of Shiatsu and acupressure. BMC Complement Altern Med, 11:88.

Röder, C., Schaefer, M., Leucht, S. (2004). Meta-Analyse zu Wirksamkeit und Verträglichkeit der Behandlung der leichten und mittelschweren Depression mit Johanniskraut. Fortschr Neurol Psychiatr, 72(6), 330-343.

Ruddy, R.R., Milnes, D. (2005). Art therapy for schizophrenia or schizophrenia-like illnesses. Cochrane Rev, 4, CD3728.

Russinova, Z., Wewiorski, N.J., Cash, D. (2002). Use of alternative health care practices by persons with serious mental illness: perceived benefits. Am I Public Health, 92(10), 1600-1603.

Saeed, S.A., Bloch, R.M., Antonacci, D.J. (2007). Herbal and dietary supplements for treatment of anxiety disorders. Am Fam Physician, 76(4), 549-556.

Saeed, S.A., Antonacci, D.J., Bloch, R.M. (2010). Exercise, yoga, and meditation for depressive and anxiety disorders. Am Fam Phys, 81(8), 981-986.

Samuels, N., Gropp, C., Singer, S.R., Oberbaum, M. (2008). Acupuncture for psychiatric illness: a literature review. Behav Med, 34(2), 55-64.

Sarris, I. (2007). Herbal medicines in the treatment of psychiatric disorders: a systematic review. Phytother Res, 21(8), 703-716.

Sarris, J., Kavanagh, D.J. (2009). Kava and St. John's Wort: current evidence for use in mood and anxiety disorders. | Altern Complement Med, 15(8), 827-836.

Sarris, I., Kavanagh, D.J., Byrne, G. (2010). Adjuvant use of nutritional and herbal medicines with antidepressants, mood stabilizers and benzodiazepines. J Psychiatr Res, 44(1), 32-41.

Sarris, I., Byrne, G.J. (2011). A systematic review of insomnia and complementary medicine. Sleep Med Rev, 15(2), 99-106.

Sarris, I., Camfield, D., Berk, M. (2011). Complementary medicine, self-help, and lifestyle interventions for Obsessive Compulsive Disorder (OCD) and the OCD spectrum: A systematic review. J Affect Disord, 138(3), 213-221.

Sarris, J., Lake, I., Hoenders, R. (2011). Bipolar disorder and complementary medicine: current evidence, safety issues, and clinical considerations. J Altern Complement Med, 17(10), 881-890.

Sarris, Mischoulon, D., Schweitzer, I. (2012). Omega-3 for bipolar disorder: meta-analyses of use in mania and bipolar depression. I Clin Psychiatry, 73(1), 81-86.

Sarris, I., Moylan, S., Camfield, D.A., Pase, M.P., Mischoulon, D., Berk, M., Jacka, F.N., Schweitzer, I. (2012). Complementary medicine, exercise, meditation, diet, and lifestyle modification for anxiety disorders: a review of current evidence. Evid Based Complement Altern Med, ID 809653.

Schneider, M., Vernon, H., Ko, G., Lawson, G., Perera, J. (2009). Chiropractic management of fibromyalgia syndrome: a systematic review of the literature. J Manipulative Physiol Ther, 32(1), 25-40.

Shelton, R.C. (2009). St.John's wort (Hypericum perforatum) in major depression. I Clin Psychiatry, 70, Suppl. 5, 23-27.

Singh, V., Singh, S.P., Chan, K. (2010). Review and meta-analysis of usage of gingko as an adjunct therapy in chronic schizophrenia. Int I Neuropsychopharmacol, 13(2), 257-271.

Smith, C.A., Hay, P.P. (2005). Acupuncture for depression. Cochrane Rev, 18 (2), CD004046.

Smith, C.A., Hay, P.P.J., MacPherson, H. (2010). Acupuncture for depression. Cochrane Rev, 1, CD004046.

Sojcher, R., Gould Fogerite, S., Perlman, A. (2012). Evidence and potential mechanisms for mindfulness practices and energy psychology for obesity and binge-eating disorder. Explore, 8(5), 271-276.

Sokhadze, T.M., Cannon, R.L., Trudeau, D.L. (2008). EEG Biofeedback as a treatment for substance use disorders: Review, rating of efficacy, and recommendations for further research. Appl Psychophysiol Biofeedback, 33(1), 1-28. 
Strauss, I.L., Coeytaux, R., McDuffie, I., Nagi, A., Williams, J.W.jr. (2011). Efficacy of complementary and alternative medicine therapies for posttraumatic stress disorder. VA-ESP Project \#09-010.

Terhorst, L., Schneider, M.J., Kim, K.H., Goozdich, L.M., Stilley, C.S. (2011). Complementary and alternative medicine in the treatment of pain in fibromyalgia: a systematic review of randomized controlled trials. I Manipulative Physiol Ther, 34(7), 483-496.

Terry, R., Perry, R., Ernst, E. (2012). An overview of systematic reviews of complementary and alternative medicine for fibromyalgia. Clin Rheumatol, 31(1), 55-66.

Thachil, A.F., Mohan, R., Bhugra, D. (2007). The evidence base of complementary and alternative therapies in depression. J Affect Disord, 97 (1), 23-35.

Tsao, I.C. (2007). Effectiveness of massage therapy for chronic, non-malignant pain: a review. Evid Based Complement Alternat Med, 4(2), 165-179.

Uebelacker, L.A., Epstein-Lubow, G., Gaudiano, B.A., Tremont, G., Battle, C.L., Miller, I.W. (2010). Hatha yoga for depression: critical review of the evidence for efficacy, plausible mechanisms of action, and directions for future research. I Psychiatr Pract, 16(1), 22-33.

Vancampfort, D., Vansteelandt, K., Scheewe, T., Probst, M., Knapen, I., De Herdt, A., De Hert, M. (2012). Yoga in schizophrenia: a systematic review of randomized controlled trials. Acta Psychiatr Scand, 126(1), 12-20.

van der Watt, G., Laugharne, I., Janca, A. (2008). Complementary and alternative medicine in the treatment of anxiety and depression. Curr Opin Psychiatry, 21, 37-42.

van Houdenhove, B., Pae, C.U., Luyten, P. (2010). Chronic fatigue syndrome: is there a role for non-antidepressant pharmacotherapy? Expert Opin Pharmacother, 11(2), 215-223.

Vickers, A.J., Cronin, A.M., Maschino, A.C., Lewith, G., MacPherson, H., Foster, N.E., Sherman, K.J., Witt, C.M., Linde, K. (2012). Acupuncture for chronic pain: individual patient data meta-analysis. Arch Intern Med, 172(19), 1444-1453.

Wang, C., Bannuru, R., Ramel, I., Kupelnick, B., Scott, T., Schmid, C.H. (2010). Tai Chi on psychological well-being: systematic review and meta-analysis. BMC Complement Altern Med, 10:23.

Wang, C.W., Chan, C.L., Ho, R.T., Tsang, H.W., Chan, C.H., Ng, S.M. (2013). The effect of qigong on depressive and anxiety symptoms: a systematic review and meta-analysis of randomized controlled trials. Evid Based Complement Alternat Med, doi: 10.1155/2013/716094.

Wang, F., Man, J.K., Lee, E.K., Wu, T., Benson, H., Fricchione, G.L., Wang, W., Yeung, A. (2013). The effects of qigong on anxiety, depression, and psychological well-being: a systematic review and meta-analysis. Evid Based Complement Alternat Med, doi: 1155/2013/152738.

Wang, H., Qi, H., Wang, B., Cui, Y., Zhu, L., Rong, Z., Chen, H. (2008). Is acupuncture beneficial in depression: A meta-analysis of 8 randomized controlled trials. | Affect Disord, 111(2-3), 125-134.

Wang, Y., Huang, L.Q., Tang, X.C., Zhang, H.Y. (2010). Retrospect and prospect of active principles from Chinese herbs in the treatment of dementia. Acta Pharmacol Sin, 31(6), 649-664.

Werneke, U., Turner, T., Priebe, S. (2006). Complementary medicines in psychiatry. Br | Psychiatry, 188, 109-121.

White, A.R., Moody, R.C., Campbell, I.L. (2007). Acupressure for smoking cessation - pilot study. BMC Complement Altern Med, 14, 7:8.

Williams, J.W., Gierisch, J.M., McDuffie, I., Strauss, J.L. (2011). An overview of complementary and alternative medicine therapies for anxiety and depressive disorders. Evidence-based Synthesis Program. www.ncbi.nih.gov/pubmed/22238805.

Wilson, R.S., Scherr, P.A., Schneider, J.A., Tang, Y., Bennett, D.A. (2007). The relation of cognitive activity to risk of developing Alzheimer's disease. Neurology, 69(20), 1911-1920.

Wittchen, H.-U., Jacobi, F. (2012). Studie zur Gesundheit Erwachsener in Deutschland. Robert-Koch-Institut/TU Dresden.

Wu, P., Fuller, C., Liu, X., Lee, H., Fan, B., Hoven, C.W., Mandell, D., Wade, C., Kronenberg, F. (2007). Use of complementary and alternative medicine among women with depression: results of a national survey. Psychiatr Serv, 58(3), 349-356.

Xia, I.,Grant, T.J. (2009). Dance therapy for schizophrenia. Cochrane Rev, 1, CD006868.

Yamakawa, I., Moriya, I., Tekeuchi, K., Nakatou, M., Motoo, Y., Kobayashi, J. (2013). Significance of kampo, Japanese traditional medicine, in the treatment of obesity: basic and clinical evidence. Evid Based Complement Alternat Med, ID 943075.

Yang, P.Y., Ho, K.H., Chen, H.C., Chien, M.Y. (2012). Exercise training improves sleep quality in middle-aged and older adults with sleep problems: a systematic review. J Physiother, 58(3), 157-163. 
Yeung, W.F., Chung, K.F., Leung, Y.K., Zhang, S.P., Law, A.C. (2009). Traditional needle acupuncture treatment for insomnia: a systematic review of randomized controlled trials. Sleep Med, 10(7), 694-704.

Zhang, Y., Han, M., Liu, Z., Wang, I., He, Q., Liu, J. (2012). Chinese herbal formula xiao yao san for treatment of depression: a systematic review of randomized controlled trials. Evid Based Complement Alternat Med, doi: 1155/2012/931636.

Zhang, Z., Chen, H., Yip, K., Ng, R., Wong, V.T. (2010). The effectiveness and safety of acupuncture therapy in depressive disorders: Systematic review and meta-analysis. J Affect Disord, 124(1-2), 9-21. 